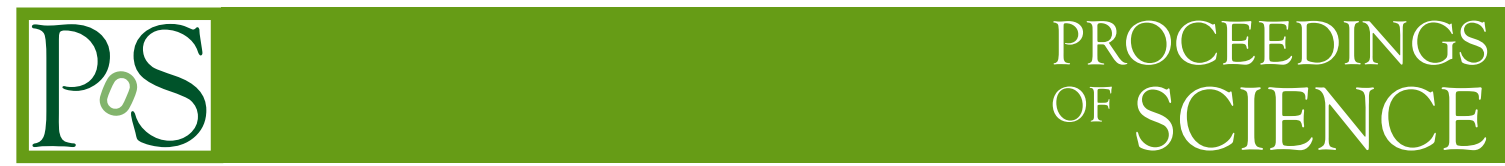

\title{
GRB 090618: a possible case of multiple GRB?
}

\section{R. Ruffini}

Dip. di Fisica, Sapienza Università di Roma and ICRA, Piazzale Aldo Moro 5, I-00185 Roma, Italy ICRANet, Piazzale della Repubblica 10, I-65122 Pescara, Italy E-mail: ruffinieicra.it

\section{Izzo, A. V. Penacchioni}

Dip. di Fisica, Sapienza Università di Roma and ICRA, Piazzale Aldo Moro 5, I-00185 Roma, Italy

E-mail: luca.izzo@icra.it, ana.penacchioni@icra.it

\section{L. Bianco, L. Caito}

Dip. di Fisica, Sapienza Università di Roma and ICRA, Piazzale Aldo Moro 5, I-00185 Roma, Italy

ICRANet, Piazzale della Repubblica 10, I-65122 Pescara, Italy

E-mail: bianco@icra.it, letizia.caitodicra.it

\section{S. K. Chakrabarti}

S. N. Bose National Center for Basic Sciences, Salt Lake, Kolkata - 700098, India Indian Center for Space Physics, Garia, Kolkata - 700084, India

\section{A. Nandi}

Indian Center for Space Physics, Garia, Kolkata - 700084, India 


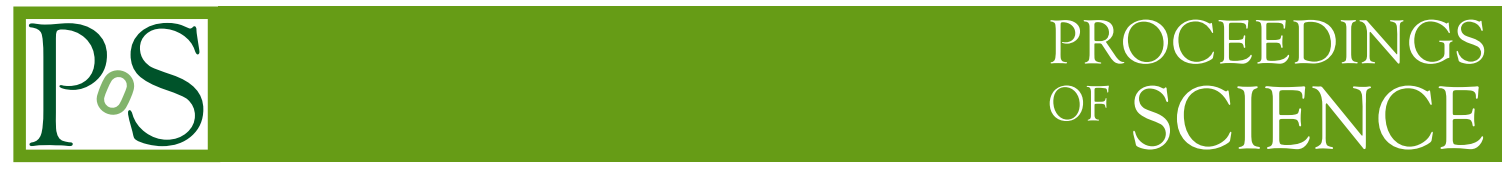

GRB 090618 is one of the nearest and most energetic GRB, at a redshift of $\mathrm{z}=0.54$, and with an isotropic energy of $E_{i s o}=2.76 \times 10^{53} \mathrm{ergs}$. We studied its temporal evolution from the data by the GBM detector on board Fermi satellite (in the energy range between $8 \mathrm{KeV}$ and $10 \mathrm{MeV}$ ) and two detectors on board Swift satellite: BAT [9] (15- $150 \mathrm{KeV})$ and XRT [10] (0.3- $10 \mathrm{KeV})$, and data from the Indian payloada RT-2/S and RT-2/G on board the Russian satellite Coronas-Photon [20]. The light curve shows a complex behavior, with several peaks. We have recently shown [22] that it is not possible to simulate the event as a single GRB in the context of the fireshell scenario [21]. We here analyze the data assuming as an ansatz that it is composed of two GRBs and we analyze them within the fireshell scenario [21]. The first, named Episode 1, lasts around $50 \mathrm{~s}$, with a dyadosphere energy of $E_{d y a}=3.87 \times 10^{52} \mathrm{erg}$. The second, named Episode 2, is compatible with a GRB of $E_{d y a}=5.0 \times 10^{53} \mathrm{erg}$. The burst is itself followed by a third signal detected in the soft $\mathrm{X}$-ray energy range. In order to perform this analysis, we have provided a detailed study of the spectral emission of all components. The results of this analysis are clearly significant for making assesment within the fireshell scenario. They are of course of general validity and can be used in order to test any possible alternative interpretation. We conclude, from the analysis of the Episode 1, that its interpretion as a separate GRB can be excluded: the interpretation of such an episode as a GRB would imply either to a P-GRB with a correct intensity but spectral properties in contrast with the observations or, alternatively, to a GRB whose spectral distribution is acceptable but with a P-GRB intensity not seen during the observation. The interpretation of the second episode as a GRB is confirmed and enriched with the identification of the P-GRB. We find very peculiar similarities between the first and the last two pulses in this second episode with respect to the relative intensity of these spikes. In conclusion, the multi component structure of GRB 090618 is confirmed. This result opens the way to a more detailed analysis for the identification of the episode 1 .

25th Texas Symposium on Relativistic Astrophysics - TEXAS 2010

December 06-10, 2010

Heidelberg, Germany 


\section{Introduction}

GRB 090618 is one of the nearest and most energetic GRB, with a redshift of $z=0.54$ [22]. It has been detected by many satellites, in different energy bands. One of these is Swift [26], launched in 2004 and still in operation. Swift is equipped with three important detectors. The Burst Alert Telescope (BAT), covering the range $15-150 \mathrm{keV}$, detects about $100 \mathrm{GRBs}$ per year, and computes the bursts positions. The X-Ray Telescope (XRT) covers the range $0.3-10 \mathrm{keV}$. It takes images of the bursts and obtains the spectra of the afterglows (afterglows are useful for higher accuracy position localizations). It also builds the light curves, later used to study the flaring and the long term decay of the afterglows. Finally, the UV/Optical Telescope (UVOT) also obtains spectra of the afterglows and follows their temporal evolution in the UV/Optical band $(170-600 \mathrm{~nm})$. From these observations, it is possible to localize the afterglow position with on-ground telescopes and determine the redshift via the individuation of common absorption lines in the corresponding spectrum. Another satellite which detected GRB 090618 is Fermi [1](formerly GLAST) launched on 11 June 2008. The mission was carried out by a collaboration among France, Germany, Italy, Japan, the United States (NASA) and Sweden. Its main instrument is the Large Area Telescope (LAT), which is used to perform an all-sky survey, in search of high energy sources. One of the detectors on board Fermi is the Gamma-ray Burst Monitor or GBM, which consists of twelve NaI and two BGO single detectors. This system is able to measure light curves and spectra of GRBs in the range $8 \mathrm{KeV}-30 \mathrm{MeV}$. In order to analyze GRB 090618 we used the data from the fourth $\mathrm{NaI}$ detector (n4), in the range $8-440 \mathrm{KeV}$ and one of the BGO detectors (b0) in the range 260 $\mathrm{KeV}-10 \mathrm{MeV}$. We made use of the Heasoft Software Tools [2] for the Fermi data. In particular, we made use of the program XSPEC [5] to fit the spectrum, using different spectral models. We also simulated the light curve and spectrum with a code which reproduces exactly the equations that describe the dynamics of the theoretical model we are considering, i.e. the fireshell (see [21]).

GRB 090618 was also detected by the Indian payloads RT-2 [20] (RT= Roentgen Telescope), on board Russian satellite Coronas-Photon [13], [14] Two detectors, namely, RT-2/S and RT-2/G consist of $\mathrm{NaI}(\mathrm{Tl}) / \mathrm{CsI}(\mathrm{Na})$ scintillators in phoswich assembly viewed by a photomultiplier tube (PMT) observed the entire light curve. RT-2/S has a viewing angle of $4^{\circ} \times 4^{\circ}$ and covers an energy range of $15 \mathrm{keV}$ to $1 \mathrm{MeV}$, whereas RT-2/G has an Al filter which sets the lower energy to $\sim 20 \mathrm{keV}$. The Mission was launched from Plesetsk Cosmodrom, Russia on January 30, 2009. During the event RT-2 payload was in the SHADOW mode (away from the Sun) during 08:16:10.207 UT and ended at 08:37:35.465 UT and the GRB060918 was detected at $77^{\circ}$ off-axis angle. During this period, the spectra was accumulated in every 100s while the eight channel count rates for each detector are accumulated every second. The entire episode was observed for a duration of more than 200 seconds.

We studied the temporal evolution of the GRB from the experimental data supported by these satellites.

\section{Analysis of data}

\subsection{Observations}

The light curve shows a multi-peak structure, with a total estimated duration of $\sim 155 \mathrm{~s}$. We 


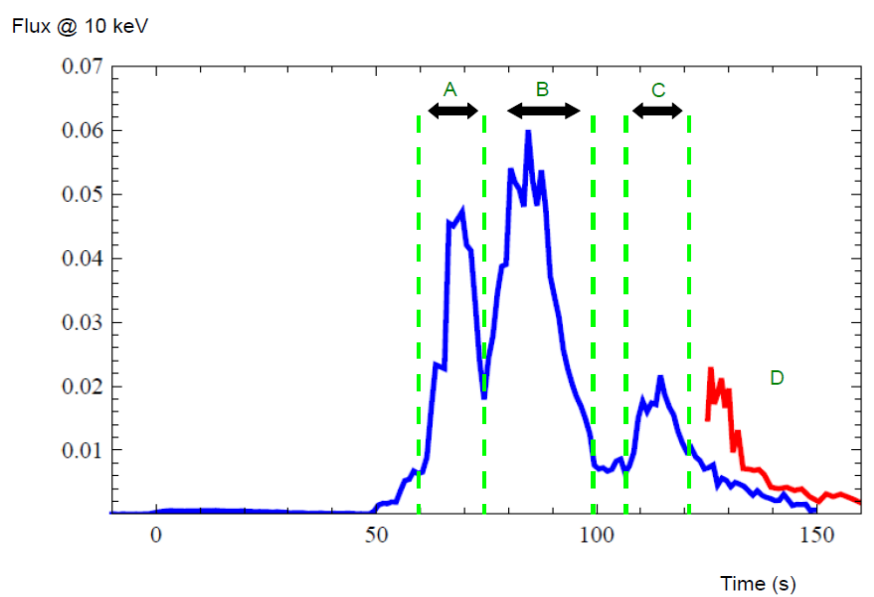

Figure 1: Flux density at $10 \mathrm{KeV}$ as detected by Fermi GBM (blue, left) and Swift XRT (red, right).

can distinguish two main episodes. The first episode, named episode 1, shows a smooth trend, ending after 50 seconds from the trigger time. The second episode, named episode 2 , shows four prominent peaks that start after 62, 69, 80 and 112 seconds after the trigger time, respectively, and each have the typical appearance of the FRED pulse [21]. We have already shown [22] that such light curve composed of two episodes, cannot be simulated as a single GRB. In view of the fact that the first emission is quite long and its spectral characteristics, we have shown that it cannot be considered as the P-GRB of the second event [22]. Since the episode 1 fulfills the Amati relation, we consider the ansatz that GRB 090618 be composed of two distinct GRBs. Therefore, we analyze the data subsequently. The episode 1 lasts around $50 \mathrm{~s}$ and should have a dyadosphere energy of $E_{d y a}=3.87 \times 10^{52}$ ergs. The episode 2 covers the period between $50 \mathrm{~s}$ and $155 \mathrm{~s}$, and it is compatible with a dyadosphere enrgy of $E_{d y a}=5 \times 10^{53}$ ergs. Interestingly, this episode 2 comprises of a third signal detected only by Swift in the soft X-ray energy range, (see Fig. 1) which appears to be the low energy tail of the second episode.

In order to test the interpretation of these two episodes, in the context of the fireshell model [21], we have performed an in-depth analysis of the spectral emission of all components. The results of this analysis are clearly significant for making assesment within the fireshell scenario. They are of course of general validity and can be used in order to test any possible alternative interpretation.

A similar analysis has been done with RT-2 data having photons in three accumulated Channels Channel 1: $15-102 \mathrm{keV}$, Channel 2: $95-250 \mathrm{keV}$ and Channel 3: $250-1000 \mathrm{keV}$. We find the most significant counts in Channel 2 with a clear evidence of the followings: (a) The first episode is 


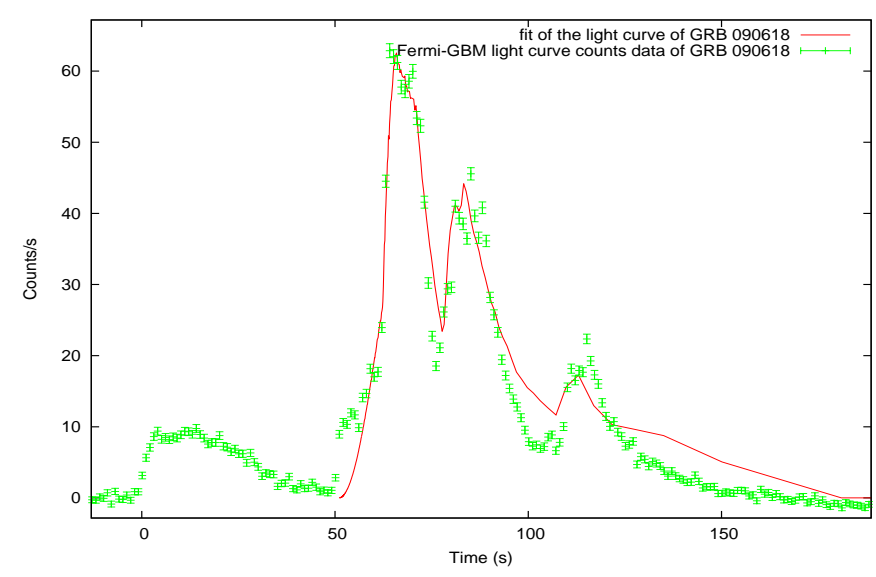

Figure 2: Count light curve of GRB 090618 obtained from Fermi NaI GBM detector, with a bin time of 1 $\mathrm{s}$. The full line represents the simulation of the second episode in the context of the fireshell scenario.

prominent and broader at higher Channels, (b) There is a clear evidence of a precursor of about 6 seconds duration before the main pulse of the second episode and (c) a clear break up into two peaks of the main pulse at intermediate energies $(35-200 \mathrm{keV})$ while at higher energies $(250-1000 \mathrm{keV})$ only the first peak of the main pulse survives. Since RT-2 data clearly show both the episodes upto $1 \mathrm{MeV}$ it compliments the results obtained by Swift (upto 150keV) and FERMI (upto 440keV) in the high and interesting range of the GRB090618. Hardness ratio plot of $(250-1000 \mathrm{keV}) /(8-250 \mathrm{keV})$ indicates that first phase of both the episodes are the hardest.

\subsection{Fireshell Scenario}

In the fireshell scenario, the GRB emission originates from a process of vacuum polarization, resulting in pair creation in the so-called dyadosphere [3]. The optically thick $e^{ \pm}$plasma has total energy $E_{\text {tot }}^{e^{ \pm}}$in the range $10^{49}-10^{54} \mathrm{ergs}$ and a temperature $\mathrm{T}$ in the range $1-4 \mathrm{MeV}$ [16]. After an early expansion, the $e^{ \pm}$-photon plasma reaches thermal equilibrium with the engulfed baryonic matter $M_{B}$ described by the dimensionless parameter $B=M_{B} c^{2} / E_{t o t}^{e^{ \pm}}$, that must be $B<10^{-2}[17$, 18]. As the optically thick fireshell composed by $e^{ \pm}$-photon-baryon plasma self-accelerates to ultra-relativistic velocities, it finally reaches the transparency condition. A flash of radiation is then emitted. This is the P-GRB[19]. The amount of energy radiated in the P-GRB is only a fraction of the initial energy $E_{t o t}^{e^{ \pm}}$. The remaining energy is stored in the kinetic energy of the optically thin baryonic and leptonic matter fireshell that, by inelastic collisions with the CBM, gives rise to a multi-wavelength emission. This is the extended afterglow. The structures observed in the prompt emission of a GRB are due to the inhomogeneities in this CBM. In this way we need few parameters for a complete description of a GRB: the dyadosphere energy $E_{d y a}$, the Baryon Loading $B$ and the CBM distribution. The afterglow presents three different regimes: a rising part, a peak and a decaying tail. We therefore define a "canonical GRB" light curve with two sharply different components : 1) the P-GRB and 2) the extended afterglow. 
The first step in the analysis of a GRB in the context of the fireshell scenario is the identification of the P-GRB. This emission is characterized by a thermal component in the spectrum which originates in the $e^{ \pm}$plasma transparency. Moreover, the P-GRB emission is just a fraction of the total energy emitted in the burst. The exact value of the energy that is released in the P-GRB emission depends on two parameters: $E_{d y a}$ and B. Since we know $E_{d y a}$, which is given by $E_{i s o}$ plus an extra contribution due to a non-electromagnetic emission (e.g. neutrino), we can obtain the exact value of $\mathrm{B}$, which will be the one corresponding to the energy observed in the P-GRB emission.

\subsection{Analysis of event 1}

We examine the episode 1 as a single GRB. We notice a peculiar structure in the first $6 \mathrm{~s}$ of emission, which shows in somewhat different from the remaining $44 \mathrm{~s}$ of the signal. We therefore attempted a first interpretation by assuming the first $6 \mathrm{~s}$ as the P-GRB component, as opposed to the remaining $44 \mathrm{~s}$ as the extended afterglow of the GRB. A value of the fit gives $E_{d y a}=3.87 \times 10^{52}$ ergs and $B=1.5 \times 10^{-4}$. This would imply a gamma Lorentz factor at the transparency $\mathrm{f} \gamma_{0}$. In turn, this value would imply [17] a spectrum of the P-GRB peaking around $\sim 200 \mathrm{keV}$, which is in contrast with the observed temperature of $58 \mathrm{keV}$. Alternatively, we have attempted a second simulation by assuming all the data as being part of the extended afterglow of a GRB. The only acceptable fit would correspond to an $E_{d y a}$ and for $B$ we selected the highest possible value of the baryon loading [18]. Even with this extreming value, assunming for the P-GRB a duration smaller than $10 \mathrm{~s}$, as confirmed from the observations of all existing P-GRBs [21], we would obtain an energy of the P-GRB of $E_{d y a}$, which is in strike contrast with the observations: such a P-GRB, with an energy flux greater than $10^{-8} \mathrm{ergs} / \mathrm{cm}^{2} / \mathrm{s}$, should be easily detectable from Fermi and Swift. Also this second possibility is not viable. We can then conclude that in no way we can interpret this episode either as a P-GRB of the second episode [22] or, as proved here, as a separate GRB.

\subsection{Analysis of event 2}

We pursue further the examination of the episode 2, already performed in a previuos work [22]. It is clearly evident in the light curve that the shape of the pulses observed in the second episode are different from the first episode: in the second episode, the pulses have the classical FRED shape, while in the first one the observed decay is smoother. In particular, we refined the data nalysis, by noticing a marked difference in spectra between the first $6 \mathrm{~s}$ of this episode and the residual emission, for $t$ larger than $56 \mathrm{~s}$. We therefore interpret the first $6 \mathrm{~s}$ as the P-GRB and the remaining data as the extended afterglow of the second episode. The best simulation of the second component is obtained for an energy of the dyadosphere $E_{d y a}=5 \times 10^{53} \mathrm{ergs}$ and for a baryon loading $B=1.5 \times 10^{-3}$, which gives a ratio between the P-GRB and the extended afterglow of 1.5 $\%$. This prediction is fully satisfied if we consider the first 6 seconds of emission of the second event as the P-GRB. From this value we obtain for the Lorentz gamma factor at the transparency the value of $\gamma_{0}=352$ and an averaged CircumBurst medium density of $\sim 1 \mathrm{part} / \mathrm{cm}^{3}$. Clearly, at this stage we have fitted with accuracy the structure of the P-GRB and the structure of the first spikes, but the last part of the GRB needs a three-dimensional analysis: at a quite large distances from the formed Black Hole, the effective area of emission of the fireshell is just a fraction of the total area. This effect is due to the lower dimensions of the blobs, with respect to the fireshell visible area, responsible for the emission. Such an analysis would be very time consuming but would not add 
anything to the understanding of the source. We have just make a general fit which allows a total energetics compatible with the observations of the remaining data.

We also plotted the flux density at $10 \mathrm{KeV}$ as detected by Fermi-GBM and Swift-XRT. We observed an extra peak that we had not observed before with GBM and decided to compare the ratio between the flux of the two first peaks with the ratio between those last two. We found that the ratios are very similar.

Finally, we computed the hardness ratio (HR) between the different bands of Swift's detectors. It is an extra tool [6] to delineate the hard-to-soft trend in this GRB, and it can be used also as an energy peak estimator for GRBs. The results of this analysis are in good agreement with two hardto-soft trends, respectively, for the two different emission episodes and, in particular, it seems to confirm a late emission extending to lower energies, of the second event.

\section{Conclusions}

The source GRB090618 is not only one of the most intense, but also one of the nearest. This gives us the very special opportunity to observe features which may be common to other GRBs, but to be unobservable due to the detector threshold effect at high redshift.

We confirmed the previous conclusion that GRB 090618 appears to be clearly composed of two episodes with very different emission properties. We have previously concluded that episode 1 could not be the P-GRB of episode 2 [22]. We have now proved combining results from various sattelites having complimentary energy sensitivity, that although Episode 1 fulfills the Amati relation, it cannot be interpreted as an independent GRB. It is to be noted that detailed RT-2 and Swift data analysis of the time lags [20] already pointed out that the origin of the gamma rays from the first episode may be totally different from that of the second episode. For instance, the time lags in the mean energy ranges of $35 \mathrm{keV}, 68 \mathrm{keV}$ and $125 \mathrm{keV}$ are more than $\sim-1 \mathrm{~s}$ for the second episode while those in the first episode are consistently less that $-2 \mathrm{~s}$, with a lag of $-7 s$ at the highest energy bin. This also points to the fact that the origins are different. We are currently examining new additional spectral features to have an understanding of this new Episode 1 component.

\section{References}

[1] Meegan, C. et al., 2009, ApJ, 702, 791

[2] http $>/ /$ heasarc.csfc.nasa.gov/heasoft

[3] Ruffini, R. arXiv: astro-ph/0106075

[4] Bianco, C. L., and Ruffini, R., 2005, ApJL, 633,13

[5] Arnaud, K., in ASP Conf. Ser., 101, Astronomical Data Analysis Software and Systems, 17

[6] Shahmoradi, A., and Nemiroff R. J., 2010, MNRAS, 407, 2075

[7] Paczynski, B., 1993, in Ann. N. Y. Acad. Sci. 688, Relativistic Astrophysics and Particle Cosmology; Sari, R., et al., 1999, ApJL, 519, 17

[8] Meszaros, P. and Rees, M. J., 1992, MNRAS, 257, 29; Sari, R., et al., 1998, ApJL, 497, 17; Zou, Y. C., et al., 2009, ApJL, 692, 92 
[9] Gehrels, N., et al., 2004, ApJ, 611, 1001

[10] Burrows, D. N. et al., 2005, Space Sci. Rev., 120, 165

[11] Evans, P. A. et. Al, 2007, MNRAS, 469, 379

[12] Rees, M.J., Ruffini R., Wheeler, J.A., Black Holes, Gravitational Waves and Cosmology: An Introduction to Current Research, 1974, Gordon and Breach Science Publishers, New York.

[13] Kotov, Yu., Kochemasov, A., Kuzin, S., Kuznetsov, V., Sylwester, J., \& Yurov, V. 2008, in 37th COSPAR Scientific Assembly

[14] Nandi, A., et al. 2009, in Proc. Int. Conf. Space Technology, ed. G. Lampropoulos \& M. Petrou, arXiv:0912.4126

[15] Nandi, A., et al. 2010, Exp. Astron., in press (arXiv:1011.3338)

[16] G. Preparata, R. Ruffini, and S. S. Xue, A\&A 338, L87-L90 (1998).

[17] R. Ruffini, J. D. Salmonson, J. R. Wilson, and S.-S. Xue, A\&A 350, 334-343 (1999).

[18] R. Ruffini, J. D. Salmonson, J. R. Wilson, and S.-S. Xue, A\&A 359, 855-864 (2000)

[19] R. Ruffini, C. L. Bianco, P. Chardonnet, F. Fraschetti, and S.-S. Xue, ApJ 555, L113-L116 (2001)

[20] A. R. Rao et al., 2011, ApJ, 728:42

[21] Ruffini et al., 2007, AIP Conf. Ser., 910, 55

[22] R. Ruffini, S. K. Chakrabarti, L. Izzo, Possible multiple components in a GRB: the case of GRB 090618, Adv. Sp. Res., in press (2011)

[23] D. Band, J. Matteson, L. Ford, et al., 1993, ApJ, 413, 281

[24] D. Guetta, E. Pian, E. Waxman, 2010, arXiv:1003.0566

[25] L. Izzo, M. G. Bernardini, C. L. Bianco, et al., 2010, JKPS, 57, 551

[26] Gehrels, N., Chincarini, G., Giommi, P., et al., 2004 ApJ, 611, 1005 